\title{
A REMARK ON EXPONENTIAL SUMS
}

\author{
BY S. K. PICHORIDES
}

\author{
Communicated by R. T. Seeley, August 29, 1976
}

ABSTRACT. The $L^{1}$ norm $\|F\|_{1}$ and the absolute value $M$ of the minimum of the real part of $F(x)=\exp \left(i_{1} x\right)+\cdots+\exp \left(i n_{N} x\right), n_{1}, \ldots, n_{N}$ distinct positive integers, satisfy the inequality $M \log M+\|F\|_{1} \geqslant$ Const $\log N$.

Let $0<n_{1}<\cdots<n_{N}$ be $N$ distinct integers, $c_{1}, \ldots, c_{N}$ complex numbers, and write

$$
F(x)=f(x)+i g(x)=c_{1} \exp \left(\operatorname{in}_{1} x\right)+\cdots+c_{N} \exp \left(\operatorname{in}_{N} x\right) .
$$

Throughout this note $C$ will denote a positive absolute constant (not always the same), integrals without limits of integration will be understood as taken over $[-\pi, \pi]$ with respect to the normalized Lebesgue measure (similarly for the corresponding norms) and $N$ will be assumed to be large.

A well-known conjecture of Hardy and Littlewood asserts that if $c_{1}=$ $\cdots=c_{N}=1$ then

$$
\|F\|_{1}>C \log N
$$

(if the $n_{i}$ 's are in arithmetic progression then we have $\|F\|_{1} \sim C \log N$ ). A method introduced by $\mathrm{P}$. Cohen and further improved by $\mathrm{H}$. Davenport and the author (see [1]) leads to the estimate

$$
\|F\|_{1}>C(\log N / \log \log N)^{1 / 2}
$$

for any $F$ with $\left|c_{i}\right| \geqslant 1, i=1, \ldots, N$. (1) appears to be the best known result up to now.

Let $M=\left|\min _{x} f(x)\right|$. Since

$$
2 M=\int(2 f+2 M) \geqslant\|2 f\|_{1}-2 M
$$

and $2 f(x) \exp \left(\operatorname{in}_{N} x\right)$ is of the same form as $F$ with $2 N$ terms, (1) implies

$$
M>C(\log N / \log \log N)^{1 / 2} \text {. }
$$

The case $c_{1}=\cdots=c_{N}=1$ of (2) has been proved by a method different than that of Cohen by K. F. Roth (see [2] where more information and references concerning these problems can be found). Again (2) appears to be the best known result concerning $M$. The example $2 f(x)=|G|^{2}-N$, where $G$ has the AMS (MOS) subject classifications (1970). Primary 42A04, 10G05; Secondary $26 \mathrm{~A} 82$. 
same form as $F$ with $n_{i+1} / n_{i}>3, i=1, \ldots, N-1$, shows that $M$ can be at least as small as $C N^{1 / 2}$.

The purpose of this note is to show that if $\left|c_{i}\right| \geqslant 1, i=1, \ldots, N$, then

$$
M \log M+\|F\|_{1}>C \log N
$$

so that either $\|F\|_{1}>C \log N$ or $M>C(\log N / \log \log N)$.

(3) is an immediate corollary of the following more general theorem whose proof although very simple is based on rather deep results of Fourier analysis.

THEOREM. If $c_{i}^{*}$ denotes the sequence $\left|c_{i}\right|$ rearranged in nonincreasing order then

$$
M \log M+\|F\|_{1}>C \sum_{i=1}^{N}\left(c_{i}^{*} / i\right)-C .
$$

Proof. The function

$$
G(z)=2 M+c_{1} z^{n_{1}}+\cdots+c_{N} z^{n_{N}}
$$

is holomorphic and its real part is not less than $M$ on the circle $|z|=1$. It follows that its real part is greater than 0 in $|z|<1$ and hence we have

$$
G(z)=|G| \exp (i q), \quad|q|=|\operatorname{Arg} G| \leqslant \pi / 2, \quad|z| \leqslant 1 .
$$

The function $f \log |G|-q g=\operatorname{Re}(F \log G)$ is harmonic and has the value 0 at the origin. An application of the mean value property for harmonic functions yields

$$
\int f \log |G|=\int q g<C\|F\|_{1}
$$

Writing $f^{-}=\max (0,-f), \log ^{+}|f|=\max (0, \log |f|)$, observing that $f=$ $|f|-2 f^{-}, \log ^{+}|f| \leqslant \log |G|$ and remembering that $\log |G|$ is harmonic with value $\log (2 M)$ at the origin we obtain

$$
\begin{aligned}
\int|f| \log ^{+}|f| & \leqslant \int|f| \log |G| \\
& \leqslant C\|F\|_{1}+2 \int f^{-} \log |G| \leqslant C\|F\|_{1}+2 M \int \log |G| \\
& =C\|F\|_{1}+2 M \log 2 M \leqslant C\left(\|F\|_{1}+M \log M+C\right) .
\end{aligned}
$$

Using now the inequality (see Remark (b)),

$$
\sum_{i=1}^{N}\left(c_{i}^{*} / i\right)<C \int|f| \log ^{+}|f|+C
$$

we obtain (4).

(3) follows immediately from (4) if we observe that $\left|c_{i}\right| \geqslant 1$ implies that the left-hand side of (5) exceeds $C \log N$.

REMARKS. (a) The argument used in the above proof is essentially the 
same as the one leading to a classical inequality of $\mathrm{M}$. Riesz for the conjugate function (see [3, Chapter VII, Theorem 2.10]).

(b) A proof of (5) can be found in [3, Chapter XII, Example 8(i)] (the result we are looking for is not stated explicitly but it is contained in the last line of the hint given there). It can also be considered as the limiting case of a better known inequality concerning rearrangements of Fourier coefficients of functions in $L^{p}$ (see [3, Chapter XII, Theorem 5.10]) and proved by the socalled extrapolation method (see [3, Chapter XII, Theorem 4.41]).

(c) A general remark concerning the above proof is that it is not based on the $L^{2}$ Parseval inequality (which is the case in [1] and [2]) but to inequalities properly belonging to the space $L \log ^{+} L$ that is a space much closer to $L^{1}$ than the space $L^{2}$. This is probably the reason that we reached now (although in a conditional form) a lower bound of the order of $\log N$ and not $(\log N)^{1 / 2}$.

\section{REFERENCES}

1. S. K. Pichorides, $A$ lower bound for the $L^{1}$ norm of exponential sums, Mathematika 21 (1974), 155-159. MR 51 \# 8048.

2. K. F. Roth, On cosine polynomials corresponding to sets of integers, Acta Arith. 24 (1973), 87-98. MR 49 \# 7221.

3. A. Zygmund, Trigonometric series, Vols. I, II, 2nd ed., reprinted with corrections and some additions, Cambridge Univ. Press, New York and London, 1968. MR 38 \# 4882.

DÉPARTMENT DE MATHÉMATIQUE, UNIVERSITÉ DE PARIS-SUD, BÂT. 425, 91405 ORSAY, FRANCE Greece

Current address: Nuclear Research Center "Demokritos", Aghia Paraskevi-Attikis, Athens, 